\title{
Basic Mechanisms in the Healing Cutaneous Wound
}

\author{
MATTHEW H. KANZLER, M.D. \\ DAVID C. GORSULOWSKY, M.D. \\ NEIL A. SWANSON, M.D.
}

\begin{abstract}
The process of wound healing can be divided into substrate, proliferative, and remodeling phases. The basic biologic and physiologic events which transpire during these stages are examined in detail. Particular emphasis is placed on the interactions between platelets, macrophages, and other inflammatory cells, and fibroblasts in the healing wound. Recent advances in the roles played by interleukins, fibronectin, and epidermal growth factor are discussed.
\end{abstract}

Production and healing of cutaneous defects are two of the most important biologic events in the practice of dermatology. A thorough understanding of the molecular events of wound healing and their subsequent biologic effects is therefore essential in both general dermatology and dermatologic surgery.

The physiologic process of wound healing can be divided temporally into three separate phases: $s u b$ strate phase, proliferative phase, and remodeling phase (Fig. 1). The substrate phase is so named because of

Matthew H. Kanzler, M.D., was Resident, Department of Dermatology, University of Michigan Medical School, Ann Arbor, Michigan. He is currently Staff Physician, Kaiser Permanente Medical Group, San Jose, California.

David C. Gorsulowsky, M.D., is Clinical Instructor, Department of Dermatology, University of California at San Francisco, and is in private practice in Fremont, California.

Neil A. Swanson, M.D., is Associate Professor, Departments of Dermatology and Otolaryngology-Head and Neck Surgery, University of Michigan Medical School.

Address reprint requests to Neil A. Swanson, M.D., Department of Dermatology, University of Michigan Medical School, 1500 E. Medical Center Drive, Ann Arbor, MI 48109 .

1156 its cellular and other interactions within the milieu of the wound which lead to preparation of the wound for subsequent events. It encompasses the first 3 to 4 days after wounding, and is characterized by vascular and inflammatory components. During this time, inflammatory cells and fibrin are exuded, imparting temporary stability to the healing wound. The proliferative phase, which occurs over the next 10 to 14 days, is dominated by proliferation of fibroblasts and the subsequent synthesis of collagen. The final phase of wound healing, the remodeling phase, takes place over a period of 6 to 12 months or longer. During this time, collagen formed during the proliferative phase is eventually replaced by a more stable form of collagen. There is a dynamic equilibrium between the synthesis of stable collagen and lysis of immature collagen. To better understand the process of wound healing, an in-depth review of these three basic phases and their interactions is in order.

\section{THE SUBSTRATE PHASE}

Immediately after injury, hemostasis is achieved by active constriction of locally damaged blood vessels, as well as aggregation of platelets to form a hemostatic plug. ${ }^{1}$ Upon exposure to biologically mature collagen and probably other connective tissue components, platelets adhere to the collagen and aggregate with each other. ${ }^{2}$ Proline and hydroxyproline are perhaps the most important determinants in recognition of collagen by platelets, as deamination or blocking of these free amino groups on the collagen molecule decreases platelet aggregation. ${ }^{3}$ However, the mechanism by which 


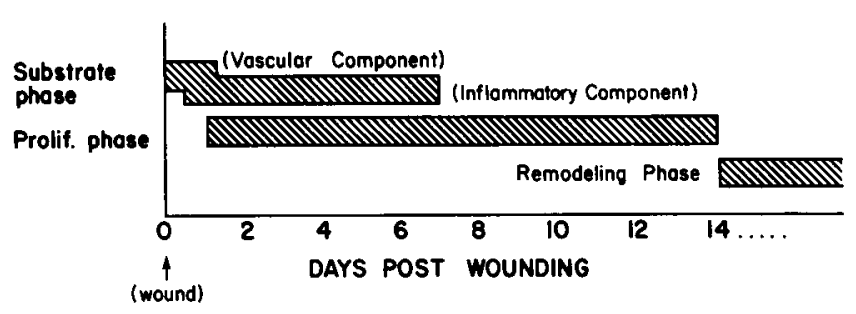

FIGURE 1. The three phases of wound healing. Note that the substrate phase encompasses both vascular and inflammatory components.

the collagen-platelet interaction takes place has not yet been well elucidated. It has been postulated but not proven that attachment of platelets to collagen is facilitated by platelet membrane fibronectin. ${ }^{4}$ It has long been known that after contacting collagen, platelets undergo transformation into activated bodies, some of which exhibit long pseudopods. ${ }^{2,3}$ Platelets possess organelles which degranulate during aggregation, releasing several factors including adenosine diphosphate (ADP), thromboxane $A_{2}$, and 5-hydroxytryptamine. These substances potentiate further aggregation of platelets augmenting formation of the vascular plug. ${ }^{1}$

A second, separate process also contributes to formation of the clot at the site of injury. Injured cells in the wound release thromboplastin (clotting factor III), which activates the extrinsic coagulation mechanism. This cascade eventually results in the cleavage of prothrombin into thrombin. Thrombin in turn cleaves fibrinogen into fibrin monomers which polymerize to form a fibrin network. ${ }^{1}$

Platelets are also active (directly and indirectly) in influencing leukocyte chemotaxis. They release proteolytic enzymes that are responsibile for initiation of the alternate complement pathway. Complement component $\mathrm{C} 5 \mathrm{a}$ is chemotactic for the cells which will be important in later events during healing. ${ }^{5}$ Leukocyte chemotaxis is probably also enhanced by 12-hydroxyeicosatetraenoic acid (12HETE) ${ }^{6}$ which is elaborated from arachidonic acid by 12 -lipoxygenase, and is released by platelets upon their contact with collagen. ${ }^{7}$ Platelet-derived 12-hydroperoxyeicosatraenoic acid (12-HPETE) and platelet activating factor induce production of leukotriene $B_{4}$, probably the major chemotactic agent, by neutrophils. ${ }^{8}$

Serum contains a platelet-derived mitogen necessary for the proliferation of fibroblast and smooth muscle cells in culture. ${ }^{9}$ This growth factor is a heatstable protein that stimulates DNA synthesis in cultured human cells. ${ }^{10} \mathrm{An}$ increase in general protein synthesis (including collagen synthesis) by the fi- broblast is also attributed to this growth factor. Although mitosis in early wound repair could be explained by this platelet-derived growth factor, continued mitogenesis 10 to 12 days later (after platelets no longer have access to the tissue) cannot be attributed to this factor. Leibovich and Ross ${ }^{11}$ have shown that activated macrophages also secrete a growth factor in vitro which stimulates fibroblast proliferation. It is not known whether this mitogen is identical or similar to the growth factor produced by platelets, but it is likely that the macrophage-derived growth factor is responsible for the continued mitogenesis later in wound healing.

Plasma contains other proteins which are important in this phase of healing. Activated Hageman factor (coagulation factor XIIa) acts through the prekallikrein-kallikrein system to cause activation of plasminogen, and generation of bradykinin. This last substance is probably active in several functions, including increased vascular permeability and leukocyte chemotaxis. In addition, factor XIIa contributes to activation of factor XI, beginning the clotting cascade. The ensuing cascade of events ultimately results in formation of thrombin. ${ }^{12}$ As in the extrinsic pathway, thrombin cleaves fibrinogen into fibrin monomers which polymerize to form a fibrin network. ${ }^{1}$ This fibrin scaffolding provides three important functions. Besides imparting temporary stability to the wound defect, the coagulum provides continued hemostasis, and a framework over which fibroblasts and epithelial cells can later migrate.

As a result of the chemotactic factors, neutrophils, the first participant cell in the inflammatory component of the substrate phase, appear in the wound at approximately six hours after wounding (Fig. 2). They reach a maximum number at 1 to 2 days and decline after approximately 2 to 3 days in

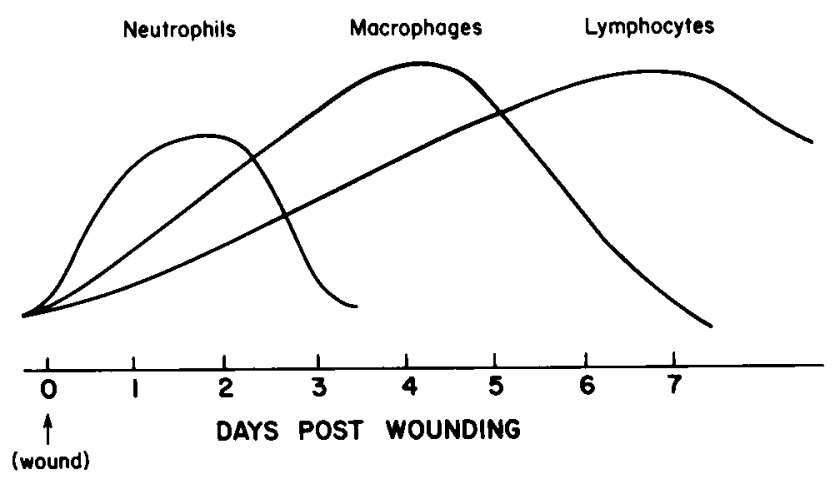

FIGURE 2. Sequence of inflammatory cells in wound healing. Neutrophils are the first cells to appear in the wound, followed by macrophages and lymphocytes. 
the absence of infection. ${ }^{13,14}$ Neutrophils do not stimulate fibroplasia, but initiate bacterial ingestion and wound debridement. Damaged polymorphonuclear leukocytes aid macrophages in debridement by releasing proteolytic, collagenolytic, and fibrinolytic enzymes into the local environment. Despite these functions, it has been shown that repair in neutropenic animals proceeds normally in the absence of infection. ${ }^{15}$

The role of lymphocytes in the process of wound healing remains controversial, although their role may be more significant than initially appreciated. Lymphocytes are at their maximum number in the wound at approximately day 6 of healing ${ }^{13}$ (Fig. 2). Perhaps the most important function of lymphocytes in wound healing is the ability of a specific subset of T-cells to synthesize lymphokines, a group of soluble humoral mediators. Two of the factors, macrophage migration inhibition factor (MIF) and macrophage activation factor (MAF), are of particular importance. MIF is a glycoprotein, molecular weight 20,000 to 40,000 , which attracts macrophages to the area of inflammation and retains them at that site. MAF activates macrophages, enhancing their various functions as described below. ${ }^{16} \mathrm{Lym}$ phocytes also release chemotactic factors for neutrophils, eosinophils, basophils, and other lymphocytes, but these factors are probably not vital to wound healing, since granulocytes appear earlier in the healing wound. Lymphokines have also been shown to stimulate fibroblast migration and proliferation, as well as collagen and noncollagen protein synthesis. ${ }^{17}$

It has recently been suggested that several previously described lymphokines are similar, if not identical substances. T-cell growth factor, thymocyte mitogenesis factor, thymocyte-stimulating factor, and killer cell helper factor are now grouped under the term interleukin $2 .{ }^{18,19}$ This 15,000 dalton protein, produced by helper T-cells, ${ }^{19}$ induces the proliferation of thymocytes after they have been stimulated by exposure to antigens. ${ }^{18}$ Analogous to the consolidation of nomenclature of lymphokines, monokines, previously known as lymphocyte-activating factor and B-cell activation factor, are now known as interleukin $1 .{ }^{19}$ Produced by macrophages, this 15,000 dalton protein has been shown to have stimulatory effects on proliferation of thymocytes and synthesis of lymphokines. ${ }^{19,20}$ In addition to its direct stimulatory effects on thymocytes, interleukin 1 probably affects proliferation of fibroblasts and granulocytes. ${ }^{18,20}$ This substance, which may be identical to the macrophage endogenous pyrogen, has also been found to promote interleukin 2 biosynthesis and secretion. Recently, it has been shown that keratinocytes produce a substance identical in composition and function to interleukin 1. This product has been named epidermal thymocyte activation factor (ETAF).

Macrophages, which are derived from circulating monocytes, are perhaps the most important inflammatory cell in wound healing. These are the only cells which can tolerate the low oxygen tensions at the leading edge of the wound. They have a relatively long life span and generally appear in the wound during the first 5 days (Fig. 2). Unlike healing in the absence of lymphocytes and neutrophils, wound repair suffers dramatically from monocytopenia. ${ }^{21}$ Migration of monocytes into the wound area may be stimulated by numerous substances, including collagen fragments, plasma, fibronectin fragments, lymphokines, bacterial endotoxins, and complement components. ${ }^{22}$ Some of these factors are components of normal tissues. Perhaps at wounding, conversion from inactive forms allows these substances to exhibit chemoattractant properties. Once macrophages are in the wound area, their activation may be initiated by lymphokines, immune complexes, complement component $\mathrm{C} 3 \mathrm{~b}$, and fibronectin, all of which are important in macrophage activation in culture. ${ }^{16,23}$

Activated macrophages perform a number of functions, the foremost of which is wound debridement. Macrophages accomplish this by production and secretion of proteinases, such as collagenases, plasminogen activators, and elastases. Activated macrophages also exhibit augmented lysosomal activity $^{24}$ and enhanced release of complement components. ${ }^{25}$ These components enhance debridement by activation of further macrophages.

Macrophages influence both collagen synthesis and lysis. ${ }^{11}$ Collagen degradation by macrophages predominates during the first 3 to 4 days of wound healing. Synthetic activity is stimulated between days 7 and 10.

The role of macrophages in neovascularization was proven experimentally by Thakral et al. ${ }^{26}$ They found that addition of macrophages to the wound on the second day after wounding-which is two to three days before these cells are seen under physiological conditions-led to ingrowth of new vessels two to three days earlier than in control circumstances.

Although eosinophils have been found to have collagenase activity, their role in wound healing is probably limited. The number of eosinophils peaks between days 7 and 14, during the period of intense collagen formation and remodeling. Therefore, they could play a role in collagen remodeling. ${ }^{27}$

Along with the intricate roles played by the vari- 
ous inflammatory cells, another substance, fibronectin, has been found to be of vital importance in wound repair. Fibronectin is localized in the clot in association with individual strands of fibrin as early as 5 days after wounding. This 440,000 dalton glycoprotein, also known as large external transformation sensitive (LETS) protein or cell surface protein (CSP), is produced by many types of differentiated cells, including fibroblasts, endothelial cells, and hepatocytes. ${ }^{28}$

Many important functions have been attributed to fibronectin. Mononuclear cells are coated by this substance, aiding in both opsonization and phagocytosis by these cells. ${ }^{28,29}$ It is also found on the surface of the cultured fibroblasts, suggesting an adhesive role between these cells and the extracellular matrix. Fibronectin probably plays a role in tissue adhesion at junctions between the epidermis and the dermis, as well as between endothelial cells and the dermis, due to its ability to cross-link with collagen and glycosaminoglycans. ${ }^{29}$ Other important processes in which fibronectin participates include blood coagulation, cellular differentiation, and production of a provisional substrate essential for cell migration and proliferation. ${ }^{30}$

Elegant work by Clark et al. ${ }^{31}$ has shown that fibronectin associated with reepithelialization is derived mainly from plasma early in wound repair, and from both plasma and fibroblasts in the later phases. They have postulated that fibroblasts migrate along the fibronectin matrix in a tractor-tread fashion. This mechanism consists of an interaction between intracellular contractile microfilaments (actin) of fibroblasts with an extracellular microfilament (fibronectin) through a plasma membrane linkage (Fig. 3). The fibroblasts actively move along the fibronectin scaffolding. Migrating epidermal cells also contain intracellular microfilaments near their inner plasma membrane, and may migrate over the fibronectin matrix in a similar fashion. ${ }^{31}$

Fibronectin is also found in normal skin. Here it is a component of the basement membane region of boin the epidermis and blood vessels. ${ }^{32}$ There is little fil-ronectin associated with papillary dermal collagen, and virtually none in the reticular dermis. $^{29}$

It appears that fibronectin has a much stronger affinity for type III collagen than for the more mature type I collagen. However, it binds strongly to all forms of denatured collagen, and this may actually account for the small proportion of type I collagen with which it interacts. Adhesion of fibroblasts to fibrin is potentiated by the cross-linkage of fibrin to fibronectin. ${ }^{29,33}$ Binding of fibronectin to denatured collagen may be important in the re-

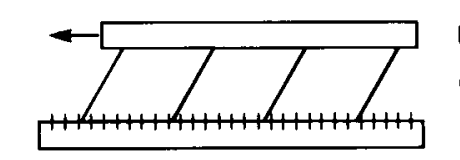

Fibroblast or epitheliol cell

Contractile (actin) microfiloments

Fibronectin microfilaments

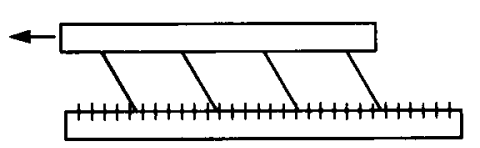

FIGURE 3. Diagrammatic representation of extracellular contractile mechanism involving fibronectin, and fibroblasts or epithelial cells. Fibroblasts and epithelial cells migrate along the fibronectin matrix in a tractor-tread fashion.

moval of collagen during remodeling, as the binding promotes phagocytosis by both fibroblasts and macrophages. ${ }^{28,34}$

\section{THE PROLIFERATIVE PHASE}

The ultimate objective of the vascular and inflammatory aspects of the substrate phase is to prepare the defect for repair. The proliferative phase, which occurs over the ensuing 10 to 14 days, results in regeneration of the epidermis, neoangiogenesis, and proliferation of fibroblasts leading to collagen synthesis.

Approximately 12 hours after wounding, epidermal cells adjacent to the wound become somewhat flattened and develop pseudopod-like extensions of their cytoplasm. ${ }^{35}$ Within 24 hours, cells 1 to $2 \mathrm{~mm}$ from the wound margin begin to migrate into the wound. Migration occurs only over viable tissue, and its rate is directly proportional to the tissueoxygen tension, being highest in hyperbaric conditions. In an optimal environment, the rate of cell movement is approximately 12 to $21 \mu \mathrm{m}$ per hour, equivalent to two to three cell diameters per hour. Epidermal cells are guided in their migration by the scaffolding of the underlying fibrin network. ${ }^{36}$ The cells burrow under crusts, if present, since a moist environment is necessary for their migration. ${ }^{35}$ They appear to move by a combination of individual cell ameboid motion and mass movement in sheets by rolling or sliding over one another. ${ }^{30}$ The desmosomes between cells at this point are smaller and fewer in number than those in resting cells. Filaments measuring 40-80 angstroms that have been shown to fix antiactin antibodies also develop at the periphery of the migrating epithelial cells. As previously stated, these filaments may align with extracellular microfilaments (fibronectin) to establish 
a tractor-tread apparatus responsible for locomotion. These contractile proteins disappear after healing, and are not present in intact normal epidermis.

Basement membrane zone changes occur beneath migrating epidermal cells. The basal lamina dissolves, and two basement membrane proteins, type IV collagen and laminin, disappear. These two basement membrane proteins mediate epithelial cell adhesion to the underlying dermis under normal circumstances. In their place, an irregular, thickened provisional matrix containing fibrin and fibronectin forms under the proliferating epidermal cells. This fibronectin complex promotes migration of fibroblasts and endothelial cells as well as migrating epidermal cells. Approximately 7 to 9 days after wounding, the basement membrane zones loses its thickened appearance, fibronectin and fibrin disappear, and type IV collagen and laminin reappear. ${ }^{37}$

Alexander has shown that in open wound margins, epidermal cells are elongated with the long axis of the cell parallel to the wound defect. He has found that the cytoplasmic organelles are polarized, with the epidermal nuclei pointing toward the open wound, which is also the direction of cell movement (Fig. 4). The Golgi complex trails, possibly secreting material which contributes to formation of the glycosaminoglycan cell coat. ${ }^{38}$ Inclusion bodies (probably phagolysosomes), as well as a prominent rough endoplasmic reticulum, also appear in the migrating epithelial cells.

Mitosis in the intact epidermis has a diurnal rhythm, being greatest during periods of inactivity. This rhythm is lost shortly after wounding, reaching a peak at approximately 48 hours, when activity may be increased 17 -fold. ${ }^{39}$ The source of the epidermal cells varies with the characteristics of the wound. In deep wounds, cells must migrate from the peripheral edges. In shallow wounds, however, reepithelialization may also occur from epidermal cells in the skin appendages, such as hair follicles. This is why healing shallow wounds may clinically appear to have buds of new epidermis.

When migrating epithelial cells meet in the center of the wound, they form desmosomal attachments and resume normal morphology and function. The rate of epithelial cell mitosis then falls to approximately three to four times the normal rate. This phenomenon, whereby epidermal cells revert to their pre-wound status, has been termed contact inhibition ${ }^{40}$ and is probably due to physical contact of cells with similar cells from the other side of the wound.

Currently under investigation is the role played

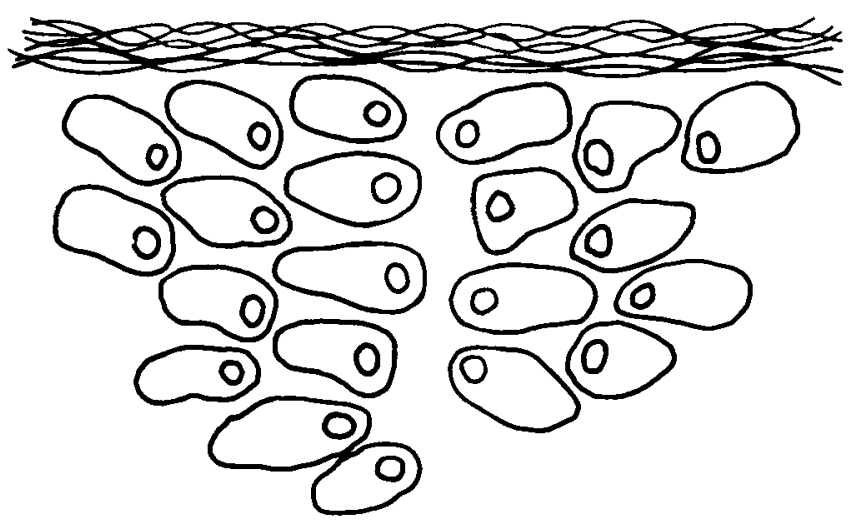

FIGURE 4. Diagrammatic representation of migrating epidermal cells in the wound. The cytoplasmic organelles are polarized, with the nucleus pointing toward the open wound, and the Golgi complex trailing.

by a heat-stable polypeptide, epidermal growth factor (EGF), with a molecular weight of approximately 15,700 in humans. The sites of synthesis of this protein factor are not known, but it has been isolated from urine, breast milk, saliva, salivary gland and amniotic fluid, and is present in epidermis. It has been shown that human EGF is biologically equivalent, and probably chemically identical to the gastric antisecretory hormone urogastrone ${ }^{41}$ In the presence of EGF, increased mitogenesis is observed in epithelial cell culture. Contact inhibition is sometimes absent in these cultures, leading to epithelial hypertrophy and hyperplasia. The mechanisms by which EGF enhances cell growth and division are unknown, although protein phosphorylation at tyrosine residues is probably an important component. ${ }^{42}$ Actions of EGF on the epidermis include increased transport of nutrients from the environment into the cell; increased activity of glycolysis; increased rate of protein, DNA, and RNA synthesis; and activation of certain enzymes such as ornithine decarboxylase. Other processes which seem to be affected by EGF include activation of protein phosphorylation, enhanced biosynthesis of prostaglandins $E_{2}$ and $F_{2 \alpha}{ }^{43}$ changes in properties of the plasma membrane, increased keratinization of the epidermis, and biosynthesis of fibronectin. ${ }^{41,44}$

EGF binds to a specific cell surface receptor, causing an increase in membrane phosphorylation. This is believed to be the initial intracellular biochemical signal generated by formation of the EGF/ receptor complex. After binding to the cell surface, the EGF/receptor complex is internalized by endocytosis in a fashion similar to that which occurs with other polypeptide hormones. The actual mechanism by which the biological responses of EGF are generated is unknown. It is assumed that the ef- 
fects are probaby due to direct interaction of EGF with the epidermis rather than through a second hormone. ${ }^{44}$

At the same time that the epidermis is regenerating, capillary buds from nearby vessels in the wound form an anastomosing network. Knighton and Hun $t^{45}$ have postulated that hypoxic conditions in the central dead space of the wound enhance the release of growth factors for macrophages, the only cell which can tolerate severe hypoxia. These growth factors are also capable of stimulating angiogenesis. When the oxygen tension increases after new vascular channels have provided blood to these dead spaces, the release of these growth factors is inhibited, in turn causing cessation of capillary growth. As the oxygen demands for collagen synthesis decrease, these newly formed vascular channels regress, leading to an avascular, cell-free scar.

As the epidermis and underlying vasculature are regenerating, changes in the dermis are also taking place which will result in repair of the dermal defect. Fibroblasts begin to appear in the wound toward the end of the inflammatory phase, beginning at 48 to 72 hours. Once this migration occurs, an additional 2 days elapse before active collagen synthesis is evident, this period being termed the "lag phase." 46 The origin of the fibroblasts is uncertain. Probably several types of mesenchymal cells are capable of differentiating into fibroblasts under appropriate conditions. Experiments with bone marrow transplants performed by Stewart et al have shown that wound fibroblasts are of local tissue origin, in contrast to the wound macrophages which are derived from hemopoietic tissue. ${ }^{47}$

Movement of fibroblasts is similar to that of epidermal cells. The fibroblasts adhere to underlying collagen and fibrin which are coated by fibronectin. As outlined earlier, the fibroblasts pull themselves along the fibronectin matrix by contraction of intracellular contractible microfilaments. Their replication is potentiated in areas of relatively low oxygen tension ( 30 to $40 \mathrm{~mm} \mathrm{Hg}$ ). Therefore, replication is greatest in front of the advancing capillary arcade in the center of the wound. As the new vessels bring increased oxygen tension to the environment, replication of fibroblasts decreases, and biosynthesis of collagen, which requires increased oxygen concentration, begins. ${ }^{48}$

The major metabolic functions of fibroblasts are the synthesis of collagen, proteoglycans, and elastin. For optimal fibroblastic activity, a mildly acidic medium is necessary. This is provided by accumulation of lactate from anaerobic metabolism in the wound. A reducing medium, provided by ascorbic acid, and oxygen tensions of at least 10 to 20 torr are also necessary. Lower oxygen levels impede formation of collagen by a decrease in oxidative metabolism. Oxygen is also required for hydroxylation, which is important in collagen synthesis.

Synthesis of collagen by fibroblasts begins by assembly of polypeptide chains at the polyribosomes. The molecular formula of each polypeptide chain can be represented as (GLY-x-y), where $x$ is frequently represented by proline and $y$ by 4 -hydroxyproline. The presence of glycine in every third position is crucial for spacial orientation of the molecule and is responsible for the left-handed helical configuration of each individual chain ${ }^{49}$ Hydroxyproline accounts for approximately $10 \%$ of the total collagen molecule. After hydroxylation, three helical chains are twisted around each other into a righthanded superhelix within the cisternae of the endoplasmic reticulum. Formation of both intra-chain and inter-chain disulfide bonds in the propeptide then occurs. ${ }^{49}$ After secretion of procollagen from fibroblasts, proteases cleave the procollagen molecule into collagen extracellularly. The collagen then self-assembles into fibrils. Finally, lysyl oxidase converts some lysine and hydroxylysine residues into aldehyde derivatives that form cross-links with similar residues in adjacent molecules, thus imparting mechanical stability to the collagen fiber. ${ }^{49}$

A prominent type of collagen formed early in the wound is type III collagen, which consists of three identical polypeptide chains. ${ }^{50}$ This form of collagen, which is associated with a gel-like consistency, first appears approximately 2 to 3 days after injury, and is maximally synthesized between days 5 and 7.

$\mathrm{O}^{\prime}$ Hare et al. ${ }^{51}$ have recently reported five factors, with presently unknown chemical natures, which have been found to stimulate the activity of prolyl hydroxylase, an important enzyme in collagen polypeptide synthesis. These collagen-stimulating factors also stimulate general protein synthesis, but to a much lesser extent. Such factors have been found to be increased in concentration significantly at day 2 when fibroblast activity first appears, and they reach a maximum concentration at day 3 , immediately prior to maximal fibroblastic activity. ${ }^{51}$ In addition, macrophages also secrete factors, including lactates, as a result of their lytic activity. These macrophage factors also have a stimulatory factor on fibroblastic activity.

Although collagen represents greater than $50 \%$ of the newly formed scar, this new connective tissue also contains glycosaminoglycans and proteoglycans. ${ }^{52,53}$ These substances are formed at the same time as collagen, with maximum synthesis at 2 weeks after wounding. The proteoglycans and glycosa- 
minoglycans function in controlling salt and water concentrations in connective tissue. These molecules may also be directly involved in cell-cell and cell-matrix interactions. ${ }^{54}$

\section{THE REMODELING PHASE}

The total amount of collagen reaches a maximum 2 to 3 weeks after wounding. Despite this, there is a progressive increase in the tensile strength of the wound for up to 1 year or more. This increase, although steady and progressive, produces a wound which is never more than $80 \%$ as strong as the original skin. In fact, at 2 weeks after wounding, when sutures are often removed, the wound has regained only $5 \%$ of its original strength, increasing to only $40 \%$ at 1 month. ${ }^{55}$

It is during this period, the remodeling phase, that the original collagen gel, synthesized in the first few weeks of wound healing, is replaced by a more stable interwoven type collagen. During this transition, type III collagen is gradually replaced by type I collagen. Water and glycosaminoglycans are lost from the wound leading to compression of the collagen fibers. Closer approximation of the fibers allows increased cross-linking by covalent bond formation, lending greater stability to the fibers. ${ }^{56} \mathrm{Re}$ modeling also results in reorientation of the fiber alignment in the scar. Those collagen fibrils which escape degradation by collagenases are generally those which are initially laid down parallel to the lines of tension.

The remodeling phase represents a dynamic equilibrium between synthesis of new, and lysis of old, collagen. Collagen resorption is tissue-specific and strictly regulated by various collagenases. Before the collagenases can exert their activity, auxiliary enzymes, such as hyaluronidase and other proteases, must expose the fibrils, removing other noncollagen substances (protein-polysaccharide complexes) which are associated with collagen in connective tissue. ${ }^{57}$

There are three types of enzymes that have the ability to digest collagen. One of these is bacterial collagenase, such as that produced by Clostridium. Another type is the group of lysosomal proteases, such as cathepsins. These proteases usually act on fragments which have previously been digested and phagocytized. Finally, and perhaps the most important enzyme in collagen degradation during wound healing, there is tissue collagenase. All tissue collagenases behave in the same general manner. They require calcium for function, as it has been shown that their action is inhibited by chelating agents such as EDTA. ${ }^{58}$

Tissue collagenases are synthesized by several cell types. In the skin, collagenase is found in the superficial layers of the papillary dermis. However, only tissues that contain epithelial cells show significant collagenolytic activity. It is therefore postulated that a mesenchymal-epithelial interaction is necessary for collagenase synthesis. Tissues rich in mesenchymal, epithelial, and inflammatory cells show the greatest synthetic activity of collagenase. ${ }^{57,58}$

Macrophages have also been observed to secrete collagenase after being activated by endotoxins or lymphokines. ${ }^{59}$ Polymorphonuclear leukocytes also produce collagenases as well as neutral proteases, both of which become significant when these cells persist, as in chronic infection. Leukocyte collagenases are not tissue-specific, and the neutral proteases are capable of digesting basement membrane material as well as collagen. Fibronectin is particularly vulnerable to the neutral proteases produced by polymorphonuclear leukocytes. These enzymes undoubtedly play a role in dehiscence of wounds during infection. ${ }^{60}$

Wound contraction, the final stage of healing, begins 1 week after wounding. This is the time that the inflammatory response is declining and connective tissue proliferation is reaching a peak. Contraction progresses at a rate of approximately 0.6 to $0.7 \mathrm{~mm} /$ day, independent of wound size. The shape of full-thickness defects does influence the rate, however. Round wounds do not contract as quickly or completely as rectangular or stellate defects. Contraction is not due to excessive deposition of collagen, as this is a rigid structural protein with no innate contractability. Also, this process can occur without significant collagen formation, as shown by experimentally inducing scurvy in animals. These animals could not synthesize new collagen, yet wound contraction continued to occur. ${ }^{61}$

The contractile forces produced by granulation tissue in wounds are derived from modified fibroblasts which contain contractile proteins. ${ }^{62}$ These fibroblasts are converted into myofibroblasts by undergoing structural, compositional, immunological, and functional changes. Electron microscopy reveals characteristics of both fibroblasts and smooth muscle cells. These myofibroblasts contain abundant rough endoplasmic reticulum due to their active collagen synthesis. Microtubules are also prominent, performing a bracing function. Myofibroblasts contain a large amount of contractile protein. In fact, granulation tissue contains as much actomyosin as an equivalent weight of smooth mus- 
cle. ${ }^{63}$ Contraction of the myofibroblasts has been shown to be inhibited by smooth muscle relaxants. Therefore, these cells share not only histologic but functional features of smooth muscle cells. Myofibroblasts are joined to one another. These cell-tocell attachments allow the myofibroblasts to pull upon one another during their migration over the neomatrix, and transmit contraction from cell to cell. These cells are distributed throughout the wound and are not concentrated at the edges of the contracting wound. ${ }^{61}$

These final processes involved in wound remodeling and contraction lead to a scar which shares many features with the preexisting normal skin, producing a functional, healed wound. It is when the orchestrated events described above are deranged or disturbed that hypertrophic or other abnormal scars are formed.

\section{SUMMARY}

Healing of cutaneous wounds is an intricately orchestrated array of events which can be divided into three phases. Important in the substrate phase are platelets and their products, complement and inflammatory cells, including lymphocytes, neutrophils, and macrophages. Activated macrophages may be the most important cells in this phase of healing, contributing to wound debridement, release of complement components, synthesis and degradation of collagen, and neoangiogenesis, among other functions.

The second phase of healing, the proliferative phase, finds epidermal cells migrating over a scaffolding of fibrin and fibronectin which has replaced the normal, thinner basement membrane zone. Epidermal growth factor is important in this step, although its exact mechanisms remain to be explained. New blood vessels supply much of the nutrition for healing at this phase. Fibroblasts are activated, forming collagen for the dermal component of the wound.

The final phase, that of remodeling, is characterized by a dynamic continuation of collagen synthesis and degradation. Degradation is accomplished by collagenase derived from several sources. As these events proceed, myofibroblasts effect wound contraction.

Wound healing is therefore a predictable, automatic chain of events eventuating in a relatively avascular scar in place of a cutaneous defect. Further manipulation of the final outcome of wound healing by the physician may be accomplished with a thorough understanding of these molecular and cellular events.

\section{REFERENCES}

1. Nossel H. Bleeding. In: Isselbacher K, Adams D, Braunwald, $E$, et al (eds), Harrison's Principles of Internal Medicine. New York, McGraw-Hill Book Co, pp 272-279, 1980, 9th ed.

2. Barnhart MI, Walsh RT, Robinson JA. A three-dimensional view of platelet responses to chemical stimuli. Ann NY Acad Sci 210:360-390, 1972.

3. Meyer FA, Frojmovic MM, Vic MM. Characteristics of the major platelet membrane site used in binding to collagen. Thromb Res 15:755-767, 1979.

4. Bensusan HB, Koh TL, Henry KG, et al. Evidence that fibronectin is the collagen receptor on platelet membranes. Proc Natl Acad Sci USA 75:5864-5868, 1978.

5. Ward P. Complement-derived leukotactic factors in pathological fluids. J Exp Med 134:109s-113s, 1971.

6. Kragballe K, Voorhees JJ. Arachidonic acid and leukotrienes in dermatology. J Invest Dermatol 81:293-296, 1983.

7. Turner S, Tainer J, Lynn W. Biogenesis of chemotactic molecules by the arachidonate lipoxygenase system of platelets. Nature 257:680-681, 1975.

8. Goldyne ME. Leukotrienes: Clinical significance. J Am Acad Dermatol 10:659-668, 1984.

9. Rutherford R, Ross R. Platelet factors stimulate fibroblasts and smooth muscle cells quiescent in plasma-serum to proliferate. J Cell Biol 69:196-203, 1976.

10. Heldin CH, Westermark B, Wasteson A. Platelet-derived growth factor: Purification and partial characterization. Proc Natl Acad Sci USA 76:3722, 1979.

11. Leibovich SJ, Ross R. A macrophage-dependent factor that stimulates the proliferation of fibroblasts in vitro. Am J Pathol 84:501-513, 1976.

12. Cochrane CG, Griffin JH. The biochemistry and pathophysiology of the contact system of plasma. Adv Immunol 33:241-306, 1982.

13. Ross R, Benditt EP. Wound healing and collagen formation. I. Sequential changes in components of guinea pig skin wounds observed in the electron microscope. J Biophys Biochem Cytol 11:677-700, 1961.

14. Ross $R$. The fibroblast and wound repair. Biol Rev 43:51-91, 1968.

15. Simpson DM, Ross R. The neutrophilic leukocyte in wound repair. A study with antineutrophil serum. J Clin Invest 51:2009-2023, 1972.

16. Ruco LP, Meltzer MS. Macrophage activation for tumor cytotoxicity induction of tumoricidal macrophages by super natants of PPD-stimulated bacillus Calmette-Guerin immune spleen cell cultures. J Immunol 119:889, 1977.

17. Wahl SM, Wahl LM, McCarthy JB. Lymphocyte-mediated activation of fibroblast proliferation and collagen production. J Immunol 121:942, 1978.

18. Gillis S. Interleukin biochemistry and biology: Summary and introduction. Fed Proc 42:2635-2639, 1983.

19. Bendtzen K. Biological properties of interleukins. Allergy 38:219-226, 1983.

20. Lachman L. Human interleukin 1: Purification and properties. Fed Proc 42:2639-2645, 1983.

21. Leibovich SJ, Ross R. The role of the macrophage in wound repair: A study with hydrocortisone and antimacrophage serum. Am J Pathol 78:71, 1975. 
22. Postlethwaite A, Kang A. Collagen and collagen peptide induced chemotaxis of human blood monocytes. J Exp Med 143:1299-1307, 1976.

23. Allison AC, Ferlugac J, Prydz H, Schorlemmer HU. The role of macrophage activation in chronic inflammation. Agents Actions 8:27-35, 1978.

24. Shavit ZB, Ray A, Goldman R. Complement and Fc receptormediated phagocytosis of normal and stimulated mouse peritoneal macrophages. Eur J Immunol 9:385, 1979.

25. Littman BH, Ruddy S. Production of the second component of complement by human monocytes: Stimulation by antigen-activated lymphocytes or lymphokines. J Exp Med 145:1344, 1979.

26. Thakral KK, Goodson WH III, Hunt TK. Stimulation of wound blood vessel growth by wound macrophages. J Surg Res 26:430-436, 1979.

27. Bassett EG, Baker JR, DeSowza P. A light microscopical study of healing incised dermal wounds in rats. With special reference to eosinophil leucocytes and to the collagenous fibres of the periwound areas. Br J Exp Pathol 58:581, 1977.

28. Grinnell F, Billingham RE, Burgess $L$. Distribution of fibronectin during wound healing in vivo. J Invest Dermatol $76: 181-189,1981$.

29. Grinnell F. Fibronectin and wound healing. Am J Dermatopathol 4:185-187, 1982.

30. Krawczyk W. Pattern of epidermal cell migration during wound healing. J Cell Biol 49:247-263, 1971.

31. Clark RAF, Winn HJ, Dvorak HF, Colvin RB. Fibronectin beneath reepithelializing epidermis in vivo: Sources and significance. J Invest Dermatol 80:026s-030s, 1983.

32. Clark RAF. Fibronectin in the skin. J Invest Dermatol 81:475-479, 1983.

33. Grinnell $F$, Feld $M$, Minter D. Cell adhesion to fibrinogen and fibrin substrata: Role of cold insoluble globulin (plasma fibronectin). Cell 19:517-525, 1980.

34. Engvall E, Ruoslahti E, Miller EJ. Affinity of fibronectin to collagens of different genetic types and to fibrinogen. J Exp Med 147:1584-1595, 1978.

35. Dillman T, Penn J. Studies on repair of cutaneous wounds. II. The healing of wounds involving loss of superficial portions of the skin. Med Proc 2:150, 1956.

36. Bullough WS, Laurence EB. Mitotic control by internal secretion: The role of the chalone-adrenaline complex. Exp Cell Res 33:176, 1964.

37. Clark RAF, Lanigan JM, DellaPelle $P$, et al. Fibronectin and fibrin providing a provisional matrix for epidermal cell migration during wound reepithelialization. J Invest Dermatol 79:264-269, 1982.

38. Alexander S. Patterns of epidermal cell polarity in healing open wounds. J Surg Res 31:456-462, 1981.

39. Christopher E. Kinetic aspects of epidermal healing. In: Maibach H, Rovee D (eds), Epidermal Wound Healing. Chicago, Year Book Medical Publíshers Inc, 1972, pp 53-70.

40. Baier R. Surface chemistry in epidermal repair. In: Maibach J, Rovee DT (eds), Epidermal Wound Healing. Chicago, Year Book Medical Publishers, Inc, 1972.

41. Carpenter G, Cohen S. Epidermal growth factor. Annu Rev Biochem 48:193-216, 1979.
42. Baldwin GS, Grego B, Hearn M. Phosphorylation of human growth hormone by the epidermal growth factor-stimulated tyrosine kinase. Proc Natl Acad Sci USA 80:5276-5280, 1983.

43. Levine L, Hassid A. Epidermal growth factor stimulates prostaglandin biosynthesis by canine kidney (MDCK) cells. Biochem Biophys Res Commun 76:1181-1187, 1977.

44. King LE, Carpenter GF. Epidermal growth factor. In: Goldsmith LA (ed), Biochemistry and Physiology of the Skin. New York, Oxford University Press, 1983, pp 269-281.

45. Knighton $E$, Hunt $T$. Regulation of wound healing angiogenesis. Surgery 90:262-270, 1981.

46. Stewart R, Duley JA, Allardyce RA. The migration of fibroblasts into an in vitro wound. Br J Exp Pathol 60:582-588, 1979.

47. Stewart R, Duley JA, Dewdney J, et al. The wound fibroblast and macrophage. II. Their origin studied in a human after bone marrow transplantation. Br J Surg 68:129-131, 1981.

48. McGrath MH, Hundahl SA. The spatial and temporal quantification of myofibroblasts. Plast Reconstr Surg 69:975-976, 1982.

49. Prockop D, Kivirikko K. Heritable diseases of collagen. N Engl J Med 311:376-386, 1984.

50. Bailey AJ, Bazin S, Sims TJ, et al. Characterization of the collagen of human hypertrophic and normal scars. Biochem Biophys Acta 21:404, 1975.

51. O'Hare RP, Fallon A, Bradley JF, et al. Isolation of collagen stimulating factors from healing wounds. J Clin Pathol 36:707-711, 1983.

52. Lamberg S1, Stoolmiller AC. Glycosaminoglycans: A biochemical and clinical review. J Invest Dermatol 63:433-449, 1974.

53. VanLis JM, Kalsbeek GL. Glycosaminoglycans in human skin. Br J Dermatol 88:355-361, 1973.

54. Silbert J. Structure and metabolism of proteoglycans and glycosaminoglycans. J Invest Dermatol 79:31s-37s, 1982.

55. Levensen SM, Geever EG, Crowley LV, et al. The healing of rat skin wounds. Ann Surg 161:293-308, 1965.

56. Bailey AJ, Bazin S, Delawney A. Changes in the nature of the collagen during development and resorption of granulation tissue. Biochem Biophys Acta 328:383-390, 1973.

57. Peacock E. Collagenolysis: The other side of the equation. World J Surg 4:297-302, 1980.

58. Grillo $\mathrm{H}$, Gross J. Collagenolytic activity during mammalian wound repair. Dev Biol 15:300, 1967.

59. Wahl LM, Wahl SM, Mergenhagen SE, Martin GR. Collagenase production by endotoxin activated macrophages. Proc Natl Acad Sci USA 71:3598, 1974.

60. McDonald JA, Baum BJ, Rosenberg DM, et al. Destruction of a major extracellular adhesive glycoprotein (fibronectin) of human fibroblasts by neutral proteases from polymorphonuclear leukocyte granules. Lab Invest 40:350-357, 1979.

61. Rudolph R. Contraction and the control of contraction. World J Surg 4:279-287, 1980.

62. Gabbiani G, Ryan GB, Majne G. Presence of modified fibroblasts in granulation tissue and their possible role in wound contraction. Experientia 27:549-550, 1971.

63. Majno G. Contraction of granulation tissue in vitro: Similarity to smooth muscle. Science 173:543-550, 1971. 